\title{
Algunas propiedades homológicas del plano de Jordan
}

\section{Some homological properties of Jordan plane}

Jonatan Andrés Gómez Parada $\mathrm{a}^{*}$ Héctor Julio Suárez Suárez b*

Fecha de Recepción: 10 - mar. - 2018.

Fecha de Aceptación: 27 - may. - 2018.

\section{Resumen}

El plano de Jordan puede ser visto como un álgebra cociente, como una extensión de Ore graduada y como una extensión PBW torcida graduada. Usando estas interpretaciones, se muestra que el plano de Jordan es un álgebra Artin-Schelter regular y Calabi-Yau torcida, además se calcula de forma explícita su automorfismo de Nakayama.

Palabras clave: Plano de Jordan, álgebras Artin-Schelter regulares, álgebras Calabi-Yau torcidas, automorfismo de Nakayama.

\begin{abstract}
The Jordan plane can be seen as a quotient algebra, as a graded Ore extension and as a graded skew PBW extension. Using these interpretations, it is proved that the Jordan plane is an Artin-Schelter regular algebra and a skew Calabi-Yau algebra, in addition its Nakayama automorphism is explicitly calculated.
\end{abstract}

Keywords: Jordan plane, Artin-Schelter regular algebras, skew Calabi-Yau algebras, Nakayama automorphism.

a Grupo de Álgebra y Análisis, Escuela de Matemáticas y Estadística, Universidad Pedagógica y Tecnológica de Colombia, Tunja.

* Correo electrónico: joangopa96@gmail.com

b Grupo de Álgebra y Análisis, Escuela de Matemáticas y Estadística, Universidad Pedagógica y Tecnológica de Colombia, Tunja.

* Correo electrónico: hector.suarez@uptc.edu.co 


\section{INTRODUCCIÓN}

Existen varias versiones sobre el origen del plano de Jordan: en [2] afirman que el plano de Jordan fue definido por primera vez en [10]; posteriormente en [3] afirman que la denominación de Jordan proviene de [5]; otros autores afirman que el plano de Jordan lo introdujo Korenskii en [13]. El plano de Jordan aparece en diferentes contextos en matemáticas y física y es uno de los ejemplos básicos en los orígenes de la geometría algebraica no conmutativa, siendo uno de los ejemplos básicos de anillos graduados no conmutativos de crecimiento cuadrático. En 1987 Artin y Schelter definieron las álgebras Artin-Schelter regulares (véase [4]) y afirmaron que el plano de Jordan (sin darle este nombre) es una de las dos álgebras regulares de dimensión global dos. Las álgebras Calabi-Yau fueron introducidas por Ginzburg en [7] y posteriormente hicieron una generalización de estas álgebras, denominadas álgebras Calabi-Yau torcidas. Las extensiones PBW torcidas fueron definidas por Gallego y Lezama en [6].

El plano de Jordan ha sido ampliamente estudiado, por ejemplo: es el álgebra de Nichols asociada a un espacio vectorial trenzado de dimensión 2 en la categoría de módulos de Yetter-Drinfeld con dimensión de Gelfand-Kirillov 2 , se conocen sus representaciones, su homología y cohomología de Hochschild, entre otras propiedades (véase por ejemplo [1, 2, 3, 12, 18]). Esta es un álgebra de Koszul (véase [23], página 208), Artin-Schelter regular (véase [4], página 172) y es un álgebra Calabi-Yau torcida que no es Calabi-Yau (véase [17]). Aunque estos resultados son ampliamente conocidos en la literatura (véase por ejemplo [4, 17, 21, 22, 24]), el objetivo de este artículo es mostrar que el Plano de Jordan satisface tales propiedades usando el hecho que tal álgebra puede presentarse como una extensión de Ore (véase [17], página 16) y como una extensión PBW torcida (véase [25], página 185). Las álgebras de Koszul, Artin-Schelter regulares, Calabi-Yau y las extensiones PBW torcidas juegan un papel importante en estudios recientes, especialmente en geometría algebraica no conmutativa (véase por ejemplo $[16,21])$.

En la sección 2 se presentan algunos preliminares y se muestra que el plano de Jordan es un álgebra cuadrática, finitamente graduada y finitamente presentada. En la sección 3 se muestra que el plano de Jordan es una extensión de Ore del anillo de polinomios $\mathbb{K}[\mathrm{x}]$ y es una extensión PBW torcida graduada biyectiva y pre-conmutativa. En la sección 4 se presenta una resolución proyectiva de $\mathbb{K}$ como A-módulo, donde A es el plano de Jordan y se demuestra que el plano de Jordan es un álgebra Artin-Schelter regular de dimensión global 2. Usando los resultados de las secciones anteriores, se muestra que en efecto el plano de Jordan es un álgebra Calabi-Yau torcida de dimensión 2 y se calcula su automorfismo de Nakayama.

\section{PRELIMINARES}

Para lo que sigue del artículo fijaremos la siguiente notación: todos los anillos tienen identidad, $\mathbb{K}$ es un cuerpo, todas las álgebras son $\mathbb{K}$-álgebras y el símbolo $\otimes$ indicará $\otimes_{\mathbb{K}}$.

Un álgebra $\mathrm{A}$ es finitamente generada como álgebra si existen elementos $a_{1}, \ldots, a_{n} \in \mathrm{A}$ tal que el conjunto $\left\{a_{i_{1}} a_{i_{2}} \cdots a_{i_{m}} \mid 1 \leq i_{j} \leq n, m \geq 1\right\}$ $\cup\{1\}$ genera a $A$ como un $\mathbb{K}$-espacio vectorial. Un álgebra $\mathrm{A}$ es $\mathbb{N}$-graduada si tiene una descomposición en $\mathbb{K}$-espacios vectoriales $\mathrm{A}=\oplus_{n \geq 0} A_{n}$ tal que $A_{i} A_{j} \subseteq A_{i+j}$, para todo $i, j \geq 0$. Se dice que A es conexa si $\mathrm{A}_{0}=\mathbb{K}$. Un elemento $x$ en $\mathrm{A}$ es homogéneo si $x \in A_{n}$ para algún $n$. Un ideal izquierdo $I$ de $A$ es llamado homogéneo o graduado si es generado por elementos homogéneos, o equivalentemente, si $I=\oplus_{n \geq 0}\left(I \cap A_{n}\right)$. En el álgebra libre $A=\mathbb{K}\left\langle x_{1}, \ldots, x_{n}\right\rangle$, la longitud de una palabra $x_{i_{1}} x_{i_{2}} \cdots x_{i_{m}}$ es $m$ y la longitud de 1 es 0 . Notemos que $\mathrm{A}$ es $\mathbb{N}$-graduada conexa, donde $\mathrm{A}_{i}$ es el $\mathbb{K}$-subespacio generado por todas las palabras de longitud $i$. Por simplicidad en la escritura, para $m \geq 0$, la suma $\oplus_{n \geq m} A_{n}$ se notará como $A_{\geq m}$.

Dada A un álgebra $\mathbb{N}$-graduada conexa finitamente generada, se dice que A es finitamente presentada si existe un ideal homogéneo $I$ generado por finitos elementos homogéneos, $I=\left(f_{1}, \ldots, f_{m}\right)$ tal que $A \cong \mathbb{K}\left\langle x_{1}, \ldots, x_{n}\right\rangle / I$. Al cociente $\mathbb{K}\left\langle x_{1}, \ldots, x_{n}\right\rangle /\left(f_{1}, \ldots, f_{m}\right)$ se le llama una presentación de A con generadores $x_{1}, \ldots, x_{n}$ y relaciones $f_{1}, \ldots, f_{m}$. Las álgebras finitamente graduadas fueron definidas por Rogalski en [21], 
un álgebra $\mathrm{A}$ es finitamente graduada si $\mathrm{A}$ es $\mathbb{N}$ $\mathbb{N}$-graduada conexa y finitamente generada como un álgebra.

Sea A un álgebra $\mathbb{N}$-graduada. Un A-módulo $\mathrm{M}$ es graduado si $\mathrm{M}$ tiene una descomposición en $\mathbb{K}$-espacios vectoriales $M=\oplus_{n \in \mathbb{Z}} M_{n}$ tal que $A_{j} M_{i} \subseteq M_{i+j}$ para todo $i \in \mathbb{Z}, j \in \mathbb{N}$. Dado un $A$-módulo graduado $M$ se define $M(i), i \in \mathbb{Z}$, como el módulo graduado que es isomorfo a $\mathrm{M}$ como un A-módulo, el cual tiene una graduación dada por $M(i)_{n}=M_{i+n}$. De forma análoga, dada un álgebra graduada $\mathrm{A}$, se define el álgebra graduada $A(i)$, con $i \in \mathbb{Z}$. Si $M y N$ son $A$-módulos, el conjunto de los $A$-homomorfismos de $M$ en $N$ se denotará como $\operatorname{Hom}_{A}(M, N)$. Si $M$ y $N$ son graduados, para un $l \in \mathbb{Z}$, se dice que $f$ es un homomorfismo l-graduado si $f\left(M_{n}\right) \subseteq N_{n+l}$ para todo $n$. Si $l=0$, se dice simplemente que $f$ es graduado.

El plano de Jordan aparece en diferentes contextos en matemáticas y física, además de presentarse como un ejemplo básico en los inicios de la geometría algebraica no conmutativa.

Definición 1. El Plano de Jordan es el álgebra libre $\mathbb{K}\langle x, y\rangle$, sujeta a la relación $y x=x y+x^{2}$, es decir, $A=\mathbb{K}\langle x, y\rangle /\left(y x-x y-x^{2}\right)$, donde $\left(y x-x y-x^{2}\right)$ es el ideal bilátero de $\mathbb{K}\langle x, y\rangle$ generado por el polinomio $y x-x y-x^{2}$.

Proposición 2. El plano de Jordan $A=\mathbb{K}\langle x, y\rangle /\left(y x-x y-x^{2}\right)$ es un álgebra finitamente graduada y finitamente presentada.

Demostración. El álgebra libre $\mathbb{K}\langle x, y\rangle$ es finitamente generada como álgebra, además de ser graduada y conexa. El ideal de relaciones $I=\left(y x-x y-x^{2}\right)$ es homogéneo de grado 2, luego el cociente -es decir, el plano de Jordanresulta finitamente generado, graduado y conexo. Como $I$ es finitamente generado, el álgebra $A$ es además finitamente presentada.

Definición 3. Si A es un álgebra finitamente generada, la dimensión de Gelfand-Kirillov (GKdim) de A se define como

$$
\operatorname{GKdim}(A)=\limsup _{n \rightarrow \infty}\left(\frac{\ln \left(\operatorname{dim}_{\mathbb{K}} V^{n}\right)}{\ln (n)}\right),
$$

donde $V$ es un $\mathbb{K}$-subespacio de dimensión finita de $A$, que genera a $\mathrm{A}$ como un álgebra y $1 \in V$.

En [14] se encuentran las propiedades básicas de la dimensión de Gelfand-Kirillov. Una de estas propiedades es la no dependencia en la elección de $V$ [14, Lema 1.1 y Lema 2.1].

El siguiente resultado es de gran utilidad para decidir si algunas álgebras son dominios o cumplen la condición de ser noetherianas. Recordemos que un elemento $x \in A$ es normal si $x A=A x$.

Lema 4 ([21], Lema 1.11). Sean A un álgebra finitamente graduada y $x \in A_{d}$ un elemento normal homogéneo, para algún $\mathrm{d} \geq 1$.

(i) Dado $x$ un elemento no divisor de cero en $A$, si A/Ax es un dominio entonces $A$ es un dominio.

(ii) $S i$ A/Ax es noetheriana, entonces $A$ es noetheriana.

Un álgebra $A=\oplus_{n \geq 0} A_{n}$ es generada en grado uno si $A$ es generada por $\mathrm{A}_{1}$. Un álgebra generada en grado uno es llamada cuadrática si el álgebra está determinada por relaciones pertenecientes a $A_{1} \otimes A_{1}$. Por lo tanto, un álgebra cuadrática A está determinada por un espacio vectorial de generadores $\mathrm{V}=\mathrm{A}_{1}$ y un subespacio arbitrario de relaciones cuadráticas $I \subset A_{1} \otimes A_{1}$.

Proposición 5. El plano de Jordan $A=\mathbb{K}\langle x, y\rangle /\left(y x-x y-x^{2}\right)$ es un álgebra cuadrática.

Demostración. Como $\mathrm{A}_{1}$ es el espacio vectorial generado por $x, y$, entonces $\mathrm{A}$ es generada en grado uno. Además, $y x-x y-x^{2} \in A_{1} \otimes A_{1}=A_{2}$, luego el plano de Jordan es un álgebra cuadrática.

\section{EXTENSIONES DE ORE Y EXTEN- SIONES PBW TORCIDAS}

En esta sección se presentan las nociones y algunas propiedades de las extensiones de Ore y de las extensiones PBW torcidas de un anillo $R$. Adicionalmente, se muestra que el plano de 
Jordan puede ser visto como una extensión de Ore y como una extensión PBW torcida del anillo de polinomios $\mathbb{K}[\mathrm{x}]$.

\subsection{Extensiones de Ore}

Se dice que A es una extensión de Ore del anillo $R$ si se cumplen las siguientes condiciones:

(i) $\mathrm{R}$ es un subanillo de A.

(ii) Existe en $\mathrm{A}$ un elemento $x$ tal que $A$ es un $R$-módulo libre a izquierda con base $\left\{x^{k}\right\}_{k \geq 0}$, es decir, cada elemento de $A$ se expresa unívocamente como una suma finita $\sum_{i} r_{i} x^{l}$, con $r_{i} \in R$.

(iii) $x r \in R x+R$, es decir, $x r=\sigma(r) x+\delta(r)$ para algunos $\sigma(r), \delta(r) \in R$, con $r \in R$.

De (iii) se obtiene que $\sigma: R \longrightarrow R$ es un endomorfismo de anillos, mientras que $\delta: R \longrightarrow R$ es una $\sigma$-derivación; es decir:

$$
\begin{aligned}
\delta(a+b) & =\delta(a)+\delta(b) \\
\delta(a b) & =\sigma(a) \delta(b)+\delta(a) b
\end{aligned}
$$

En particular, $\sigma(1)=1$ y $\delta(1)=0$. La extensión de Ore del anillo $R$ se nota por $A=R[x ; \sigma, \delta]$.

Proposición 6. El plano de Jordan $A=\mathbb{K}\langle x, y\rangle /\left(y x-x y-x^{2}\right)$ es una extensión de Ore del anillo de polinomios $\mathbb{K}[x]$.

Demostración. Sean $\sigma: \mathbb{K}[x] \longrightarrow \mathbb{K}[x]$, con $\sigma=\mathrm{id}_{\mathbb{K}[x]}$, y $\delta: \mathbb{K}[x] \longrightarrow \mathbb{K}[x]$, tal que

$$
\delta\left(\sum a_{i} x^{i}\right)=\sum i a_{i} x^{i+1} .
$$

Sean $f, g \in \mathbb{K}[x]$, entonces $f=\sum a_{i} x^{i}$ y $g=$ $\sum b_{i} x^{i}$, así

$$
\begin{aligned}
\delta(f+g) & =\delta\left(\sum a_{i} x^{i}+\sum b_{i} x^{i}\right) \\
& =\delta\left(\sum\left(a_{i}+b_{i}\right) x^{i}\right) \\
& =\sum i\left(a_{i}+b_{i}\right) x^{i+1} \\
& =\sum i a_{i} x^{i+1}+\sum i b_{i} x^{i+1} \\
& =\delta(f)+\delta(b) .
\end{aligned}
$$

$$
\begin{aligned}
\delta(f g)= & \delta\left(\sum_{t}\left(\sum_{i=0}^{t} a_{i} b_{t-i}\right) x^{t}\right) \\
= & \left(\sum_{t} t\left(\sum_{i=0}^{t} a_{i} b_{t-i}\right) x^{t+1}\right) \\
= & \left(\sum_{t}\left(\sum_{i=0}^{t} t a_{i} b_{t-i}\right) x^{t+1}\right) \\
= & \left(\sum_{t}\left(\sum_{i=0}^{t}(t-i) a_{i} b_{t-i}\right) x^{t+1}\right) \\
+ & \left(\sum_{t}\left(\sum_{i=0}^{t} i a_{i} b_{t-i}\right) x^{t+1}\right) \\
= & \left(\sum a_{i} x^{i}\right)\left(\sum i b_{i} x^{i+1}\right) \\
& +\left(\sum i a_{i} x^{i+1}\right)\left(\sum b_{i} x^{i}\right) \\
= & f \delta(g)+\delta(f) g \\
= & \sigma(f) \delta(g)+\delta(f) g .
\end{aligned}
$$

luego, $\delta$ es una $\sigma$-derivación.

Sea $A=\mathbb{K}\langle x, y\rangle /\left(y x-x y-x^{2}\right)$, si consideramos en A el elemento $y x-x y-x^{2}=0$, se tiene que $y x=x y+x^{2}$. Si $\sigma, \delta: \mathbb{K}[x] \longrightarrow \mathbb{K}[x]$ son como antes, entonces $\sigma(x)=x$ y $\delta(x)=x^{2}$ y así $y x=\sigma(x) y+\delta(x)$.

Sea $n \in \mathbb{N}$ tal que $y x^{n}=\sigma\left(x^{n}\right) y+\delta\left(x^{n}\right)$, es decir $y x^{n}=x^{n} y+n x^{n+1}$, entonces,

$$
\begin{aligned}
y x^{n+1} & =\left(y x^{n}\right) x \\
& =\left(x^{n} y+n x^{n+1}\right) x \\
& =x^{n} y x+n x^{n+2} \\
& =x^{n}\left(x y+x^{2}\right)+n x^{n+2} \\
& =x^{n+1} y+x^{n+2}+n x^{n+2} \\
& =x^{n+1} y+(n+1) x^{n+2} \\
& =\sigma\left(x^{n+1}\right) y+\delta\left(x^{n+1}\right) .
\end{aligned}
$$

Por tanto, para $f=\sum a_{i} x^{i} \in \mathbb{K}[x]$, se tiene que:

$$
\begin{aligned}
y f & =y \sum a_{i} x^{i} \\
& =\sum a_{i} y x^{i} \\
& =\sum a_{i}\left(x^{i} y+i x^{i+1}\right)
\end{aligned}
$$




$$
\begin{aligned}
& =\sum a_{i} x^{i} y+\sum a_{i} i x^{i+1} \\
& =\left(\sum a_{i} x^{i}\right) y+\sum i a_{i} x^{i+1} \\
& =\sigma\left(\sum a_{i} x^{i}\right) y+\delta\left(\sum a_{i} x^{i}\right) \\
& =\sigma(f) y+\delta(f) .
\end{aligned}
$$

Por lo anterior se puede concluir que:

(i) $\mathbb{K}[x] \hookrightarrow \mathbb{K}\langle x, y\rangle /\left(y x-x y-x^{2}\right)$.

(ii) Existe en $\mathbb{K}\langle x, y\rangle /\left(y x-x y-x^{2}\right)$ un elemento y tal que $\mathbb{K}\langle x, y\rangle /\left(y x-x y-x^{2}\right)$ es un $\mathbb{K}[x]$ módulo libre a izquierda con base $\left\{y^{k}\right\}_{k \geq 0}$.

(iii) $\quad y r \in \mathbb{K}[x] y+\mathbb{K}[x], \quad$ es decir, $\quad y r=$ $\sigma(r) y+\delta(r)$, para algunos $\sigma(r), \delta(r) \in \mathbb{K}[x]$, con $r \in \mathbb{K}[x]$.

Con lo cual,

$$
\mathbb{K}\langle x, y\rangle /\left(y x-x y-x^{2}\right) \cong \mathbb{K}[x][y ; \sigma, \delta] .
$$

\subsection{Extensiones PBW torcidas}

Definición 7. Sean $R$ y $A$ dos anillos. Se dice que $A$ es una extensión PBW (Poincaré-Birkhoff-Witt) torcida de $R$ si cumple las siguientes condiciones:

(i) $R \subseteq A$;

(ii) Existen elementos $x_{1}, \ldots, x_{n} \in A$ tal que $A$ es un $R$-módulo libre a izquierda, con base el conjunto $\operatorname{Mon}(A)$ de los monomios estándar,

$$
\begin{gathered}
\operatorname{Mon}(A):=\left\{x^{\alpha}:=x_{1}^{\alpha_{1}} \cdots x_{n}^{\alpha_{n}} \mid \alpha=\right. \\
\left.\left(\alpha_{1}, \ldots, \alpha_{n}\right) \in \mathbb{N}^{n}\right\} .
\end{gathered}
$$

(iii) Para cada $1 \leq i \leq n$ y cada $r \in R \backslash\{0\}$, existe un elemento $c_{i, r} \in R \backslash\{0\}$ tal que $x_{i} r-c_{i, r} x_{i} \in R$. que

(iv) Para $1 \leq i, j \leq n$ existe $c_{i, j} \in R \backslash\{0\}$ tal

$$
x_{j} x_{i}-c_{i, j} x_{i} x_{j} \in R+R x_{1}+\cdots+R x_{n} .
$$

Bajo estas condiciones $A$ se denota como $\mathrm{A}=\sigma(R)\left\langle x_{1}, \ldots, x_{n}\right\rangle$. También denotaremos $|\alpha|:=\alpha_{1}+\alpha_{2}+\cdots+\alpha_{n}$.
Proposición 8 ([6], Proposición 3). Sea $A$ una extensión PBW torcida de R. Para cada $1 \leq i \leq n$, existe un endomorfismo inyectivo $\sigma_{i}: R \longrightarrow R y$ una $\sigma_{i}$-derivación $\delta_{i}: R \longrightarrow R$ tal que

$$
x_{i} r=\sigma_{i}(r) x_{i}+\delta_{i}(r), \quad r \in R .
$$

Definición 9 ([25], Definición 2.3). Sea A una extensión PBW torcida de $R, \sum:=\left\{\sigma_{1}, \ldots, \sigma_{n}\right\}$ y $\Delta:=\left\{\delta_{1}, \ldots, \delta_{n}\right\}$, donde $\sigma_{i}$ y $\delta_{i}(1 \leq i \leq n)$ son como en la proposición anterior.

(a) A es llamada pre-conmutativa si la condición (iv) en la Definición 7 es reemplazada por: para cualesquiera $1 \leq i, j \leq n$, existe $c_{i, j} \in R \backslash\{0\}$ tal que

$$
x_{j} x_{i}-c_{i, j} x_{i} x_{j} \in R x_{1}+\cdots+R x_{n} .
$$

(b) A es llamada cuasi-conmutativa si las condiciones (iii) y (iv) en Definición 7 son reemplazadas por

(iii') para cada $1 \leq i \leq n$ y todo $r \in R \backslash\{0\}$, existe $c_{i, j} \in R \backslash\{0\}$ tal que

$$
x_{i} r=c_{i, r} x_{i}
$$

(iv') para cualesquiera $1 \leq i, j \leq n$, existe $c_{i, j} \in$ $R \backslash\{0\}$ tal que

$$
x_{j} x_{i}=c_{i, j} x_{i} x_{j}
$$

(c) $A$ es llamada biyectiva si $\sigma_{i}$ es biyectivo para cada $\sigma_{i} \in \sum$ y $c_{i, j}$ es invertible para cada $1 \leq$ $i<j \leq n$.

(d) $\mathrm{Si} \sigma_{i}=\mathrm{id}_{R}$ para cada $\sigma_{i} \in \sum$, se dice que $A$ es una extensión PBW torcida de tipo derivación.

(e) Si $\delta_{i}=0$ para todo $\delta_{i} \in \Delta$, se dice que $A$ es una extensión PBW torcida de tipo endomorfismo.

(f) Un elemento $r$ de $R$ tal que $\sigma_{i}(r)=$ $=r$ y $\delta_{i}(r)=0$ para todo $1 \leq i \leq n$ será llamado una constante. $A$ es llamada constante si todo elemento de $R$ es constante.

(g) A es llamada semi-conmutativa si $A$ es cuasi-conmutativa y constante. 
Estas extensiones y sus subclases han sido bastante estudiadas recientemente (véase por ejemplo $[6,8,16,19,22,23,24,25])$.

\subsection{El plano de Jordan como una extensión PBW torcida}

Proposición 10. El plano de Jordan es una extensión PBW torcida biyectiva y pre-conmutativa.

Demostración. Considérese el plano de Jordan $A=\mathbb{K}\langle x, y\rangle /\left(y x-x y-x^{2}\right)$, entonces se tiene que:

(i) $\mathbb{K}[x] \subset A$.

(ii) Existe y $\in A$ tal que $A$ es un $\mathbb{K}[x]$-módulo con base $\left\{y^{n} \mid n \in \mathbb{N}\right\}$.

(iii) Como en $A$ se tiene que $y x-x y-x^{2}=0$, entonces $y x-x y=x^{2} \in \mathbb{K}[x]$.

Por tanto el plano de Jordan puede expresarse como una extensión PBW torcida, es decir,

$$
\mathbb{K}\langle x, y\rangle /\left(y x-x y-x^{2}\right) \cong \sigma(\mathbb{K}[x])\langle y\rangle .
$$

Además, como $y x=x y+x^{2}$, se debe tener que para el endomorfismo $\sigma$ y la $\sigma$-derivación $\delta$ de la Proposición $8, \sigma(x)=x$ y $\delta(x)=x^{2}$, coincidiendo con el automorfismo $\sigma$ y la $\sigma$-derivación $\delta$ del plano de Jordan como extensión de Ore del anillo $\mathbb{K}[x]$. Así, se puede concluir que el plano de Jordan es una extensión PBW torcida biyectiva y pre-conmutativa.

\subsection{Extensiones PBW torcidas graduadas}

Como una generalización de las extensiones de Ore iteradas graduadas, en [22] fueron definidas las extensiones PBW torcidas graduadas.

Proposición 11 ([22], Proposición 2.7). Sea $R=\oplus_{m \geq 0} R_{m}$ un álgebra $\mathbb{N}$-graduada y sea $A=\sigma(R)\left\langle x_{1}, \ldots, x_{n}\right\rangle$ una extensión PBW torcida biyectiva de $R$ que satisface las siguientes condiciones:

(i) $\sigma_{i}$ es un homomorfismo de anillos graduados y $\delta_{i}: R(-1) \longrightarrow R$ es una $\sigma_{\boldsymbol{i}}$ - derivación para todo $1 \leq i \leq n$, donde $\sigma_{i} y \delta_{i}$ son como en la Proposición 8.

(ii) $x_{j} x_{i}-c_{i, j} x_{i} x_{j} \in R_{2}+R_{1} x_{1}+\cdots+R_{1} x_{n}$, como en (1) $y c_{i, j} \in R_{0}$.

Para $p \geq 0$, sea $A_{p}$ el $\mathbb{K}$-espacio generado por el conjunto

$$
\left\{r_{t} x^{\alpha}|\quad t+| \alpha \mid=p, r_{t} \in R_{t}, x^{\alpha} \in \operatorname{Mon}(A)\right\} .
$$

Entonces $A$ es un álgebra $\mathbb{N}$-graduada con graduación

$$
A=\bigoplus_{p \geq 0} A_{p}
$$

Definición 12. Sea $A=\sigma(R)\left\langle x_{1}, \ldots, x_{n}\right\rangle$ una extensión PBW torcida biyectiva de un álgebra $\mathbb{N}$-graduada $R=\oplus_{m \geq 0} R_{m}$. Se dice que $A$ es una extensión PBW torcida graduada si satisface las condiciones (i) y (ii) en la Proposición 11.

Proposición 13. El plano de Jordan es una extensión PBW torcida graduada.

Demostración. Por la Proposición 10 tenemos que el plano de Jordan $A=\mathbb{K}\langle x, y\rangle /\left(y x-x y-x^{2}\right)$ es una extensión PBW torcida biyectiva $\mathrm{y}$ pre-conmutativa de $\mathbb{K}[x]$, que resulta ser un álgebra $\mathbb{N}$-graduada, con la graduación usual dada por el grado de los monomios. Además, el endomorfismo $\sigma$ y la $\sigma$-derivación $\delta$ de la Proposición 8 están dados por

$$
\sigma=\operatorname{id}_{\mathbb{K}[x]}, \quad \delta\left(\sum a_{i} x^{i}\right)=\sum i a_{i} x^{i+1} .
$$

Luego, $\sigma$ es un homomorfismo de anillos graduados y $\delta: \mathbb{K}[x](-1) \longrightarrow \mathbb{K}[x]$ es una $\sigma$-derivación. Por tanto, $A=\mathbb{K}\langle x, y\rangle /\left(y x-x y-x^{2}\right)$ es una extensión PBW torcida graduada de $\mathbb{K}[x]$.

\section{4. ÁLGEBRAS ARTIN-SCHELTER RE- GULARES VERSUS ÁLGEBRAS CALA- BI-YAU TORCIDAS}

Reyes, Rogalski y Zhang en [20, Lema 1.2] demostraron que en el caso de álgebras graduadas conexas, las álgebras Calabi-Yau torcidas y las álgebras Artin-Schelter regulares coinciden. 


\section{1 Álgebras Artin-Schelter regulares}

Las álgebras Artin-Schelter regulares son una clase de álgebras graduadas introducidas por Artin y Schelter en [4]. En tal artículo los autores clasificaron las álgebras Artin-Schelter regulares generadas en grado uno y de dimensión global menor o igual a 3 .

Definición 14. Sea $A=\mathbb{K} \oplus A_{1} \oplus A_{2} \oplus \cdots$ un álgebra graduada finitamente presentada. El álgebra $A$ se dice Artin-Schelter (AS) regular si satisface:

(i) $A$ tiene dimensión global finita $d$, es decir, todo $A$-módulo graduado tiene dimensión proyectiva menor o igual a $d$.

(ii) $A$ tiene dimensión de Gelfand-Kirillov finita.

(iii) $A$ es Gorenstein, es decir, $\operatorname{Ext}_{A}^{q}(\mathbb{K}, A)=0$ si $q \neq d$ y $\operatorname{Ext}_{A}^{d}(\mathbb{K}, A) \cong \mathbb{K}$.

En la definición anterior se considera a $\mathbb{K}$ como un $A$-módulo, de la siguiente manera: dado $a \in A$, $a=k_{0}+a_{d_{1}}+\cdots a_{d_{r}}$, con $k_{0} \in \mathbb{K}$ y $a_{d_{i}} \in A_{d_{i}}$. Entonces para $k \in \mathbb{K}$ y $a \in A$ se define $a \cdot k:=k_{0} k$.

Sea $A$ como en la definición anterior. Podemos definir el homomorfismo canónico de $A$-módulos $j: A \rightarrow \mathbb{K}, \operatorname{con} j(a):=k_{0}$ para cada a $a \in \mathrm{A}$.

Proposición 15 ([4], Página 173). La sucesión de A-módulos

$$
A^{r_{2}} \stackrel{h_{M}}{\longrightarrow} A^{r_{1}} \stackrel{h_{x}}{\longrightarrow} A \stackrel{j}{\longrightarrow} A \mathbb{K} \longrightarrow 0
$$

es exacta.

A continuación se presentan dos ejemplos de sucesiones exactas de $A$-módulos del cuerpo $\mathbb{K}$ como $A$-módulo, las cuales serán de utilidad para mostrar ejemplos de álgebras Artin-Schelter regulares.

Ejemplo 16. Considérese la $\mathbb{K}$-álgebra $A=\mathbb{K}\langle x, y\rangle$ sujeta a la relación $x y=q y x$, con $q \in \mathbb{K}^{*}$, es decir, $A=\mathbb{K}\langle x, y\rangle /(x y-q y x)$ (A es conocida como el plano cuántico). Entonces $r_{1}=2$, y $r_{2}=1$, con $f_{1}=x y-q y x$.

$$
M=\left[\begin{array}{ll}
-q y & x
\end{array}\right], \quad \mathbf{x}=\left[\begin{array}{l}
x \\
y
\end{array}\right]
$$
Sean $h_{M}(a)=a\left[\begin{array}{ll}-q y & x\end{array}\right]$ y $h_{\mathbf{x}}(b, c)=[b c]\left[\begin{array}{l}x \\ y\end{array}\right]$
para todo $a, b, c \in A$. Por tanto

$$
{ }_{A} A \stackrel{h_{M}}{\longrightarrow} A^{2} \stackrel{h_{\mathbf{x}}}{\longrightarrow} A \stackrel{j}{\longrightarrow} \mathbb{K}_{A} \longrightarrow 0
$$

es una sucesión exacta de $A$-módulos proyectivos de $\mathbb{K}$ como $A$-módulo. Además dado que $A / A x \cong \mathbb{K}[y]$ es un dominio noetheriano, por el Lema 4 se tiene que $A$ es un dominio noetheriano y por tanto $h_{M}$ resulta ser inyectiva, con lo cual

$$
0 \longrightarrow_{A} A \stackrel{h_{M}}{\longrightarrow} A^{2} \stackrel{h_{\mathbf{x}}}{\longrightarrow} A \stackrel{j}{\longrightarrow} \mathbb{K}_{A} \longrightarrow 0
$$

es una resolución proyectiva de $\mathbb{K}$ como $A$-módulo.

Ejemplo 17. Sea $A=\mathbb{K}\langle x, y\rangle /\left(y x-x y-x^{2}\right)$ el plano de Jordan. Entonces $r_{1}=2$ y $r_{2}=1$, con $f_{1}=y x-x y-x^{2}$.

$$
M=\left[\begin{array}{ll}
y-x & -x
\end{array}\right], \quad \mathbf{x}=\left[\begin{array}{l}
x \\
y
\end{array}\right] .
$$

Por tanto

$$
{ }_{A} A \stackrel{h^{\prime}}{\longrightarrow}{ }_{A} A^{2} \stackrel{g^{\prime}}{\longrightarrow}{ }_{A} A \stackrel{j}{\longrightarrow} \text { } \mathbb{K} \longrightarrow 0
$$

es una sucesión exacta de $A$-módulos proyectivos de $\mathbb{K}$ como $A$-módulo, donde $h^{\prime}(a)=a$ $\left[\begin{array}{ll}y-x & -x\end{array}\right]$ y $g^{\prime}(b, c)=\left[\begin{array}{ll}b & c\end{array}\right]\left[\begin{array}{l}x \\ y\end{array}\right]$, para todo a, b,
$\mathrm{c} \in A$.

Análogo al plano cuántico, $A / A x \cong \mathbb{K}[y]$ es un dominio noetheriano, luego $h^{\prime}$ resulta ser inyectiva, y por tanto

$$
0 \longrightarrow_{A} A \stackrel{h^{\prime}}{\longrightarrow} A^{2} \stackrel{g^{\prime}}{\longrightarrow} A \stackrel{j}{\longrightarrow} A \mathbb{K} \longrightarrow 0
$$

es una resolución proyectiva de $\mathbb{K}$ como $A$-módulo.

El cálculo hecho en el Ejemplo 17 es similar al realizado en [21, Ejemplo 1.25], salvo que la resolución allí obtenida es de módulos libres graduados. 
Sea $A$ un álgebra finitamente graduada y $M$ un $A$-módulo graduado acotado a izquierda, es decir, $M_{n}=0$ para $n \ll 0$. Sea $\left\{m_{i}\right\} \subseteq M$ un conjunto de elementos homogéneos que generan a $M$ minimalmente como $A$-módulo. Entonces, existe un $A$-homomorfismo graduado sobreyectivo $\phi: \bigoplus_{i} A\left(-d_{i}\right) \rightarrow M$, con $d_{i}:=\operatorname{deg}\left(m_{i}\right)$ (i. e., $m_{i}$ es un elemento homogéneo de grado $d_{i}$. En tal caso $\phi$ es llamada una sobreyección minimal de un módulo libre graduado sobre $M$.

Una resolución libre graduada de un $A$-módulo $M$ de la forma

$$
\begin{gathered}
\cdots \bigoplus_{i} A\left(-a_{n, i}\right) \stackrel{d_{n-1}}{\longrightarrow} \cdots \stackrel{d_{2}}{\longrightarrow} \bigoplus_{i} A\left(-a_{2, i}\right) \\
\stackrel{d_{1}}{\longrightarrow} \bigoplus_{i} A\left(-a_{1, i}\right) \stackrel{\varepsilon}{\longrightarrow} M \longrightarrow 0
\end{gathered}
$$

es llamada minimal si cada $d_{i}$ es una sobreyección minimal en $\operatorname{Im}\left(d_{i}\right)$ para todo $i \geq 1$ y $\varepsilon$ : $\bigoplus_{i} A\left(-a_{1, i}\right) \longrightarrow M$ es una sobreyección minimal sobre M (véase [21, Página 9]).

Proposición 18. ([21], Lema 1.24) Una resolución libre graduada $P$. de $M$ es minimal si y solo si para todo $i \geq 1$ se tiene que $\operatorname{Im}\left(d_{i}\right) \subseteq A_{\geq 1} P_{i}$.

Definición 19. Sean A un álgebra finitamente graduada y $M, N A$-módulos Z-graduados.

- $\operatorname{Hom}_{g r-A}(M, N):=\{f: M \rightarrow N \mid f$ es un A-homomorfismo graduado $\}$.

$$
\text { - } \underline{\operatorname{Hom}}_{A}(M, N)=\bigoplus_{d \in \mathbb{Z}} \operatorname{Hom}_{g r-A}(M, N(d)) \text {. }
$$

- $\underline{\operatorname{Ext}}_{A}^{i}(M, N)$ es definido tomando un resolución libre graduada de $M$, aplicando el funtor $\underline{\operatorname{Hom}}_{A}(-, N)$ y calculando la $i$-ésima homología.

En la definición anterior $\operatorname{Hom}_{g r-A}(M, N)$ es un grupo abeliano; más aún, si $f \in \operatorname{Hom}_{g r-A}(M, N)$ y $k \in \mathbb{K}$, entonces se define $(k \cdot f)(m):=k \cdot f(m)$, para $m \in M$, con lo cual $\operatorname{Hom}_{g r-A}(M, N)$ resulta ser un $\mathbb{K}$-espacio vectorial.

Sean $S:=\underline{\operatorname{Hom}}_{g r-A}(M, N)$ y $S_{d}:=\operatorname{Hom}_{g r-A}$ $(M, N(d))$, para cada $d \in \mathbb{Z}$, fácilmente se muestra que la colección $\left\{S_{d}\right\}_{d \in \mathbb{Z}}$ define una graduación de $S$.
Observación 20 ([21], Página 10). Sean A un álgebra finitamente graduada $A$ y $M, N A$-módulos Z-graduados.

(i) Existe una inclusión natural $\underline{\operatorname{Hom}}_{A}(M, N) \hookrightarrow \operatorname{Hom}_{A}(M, N)$.

(ii) Si $M$ es finitamente generado, entonces

$$
\begin{aligned}
& \underline{\operatorname{Hom}}_{A}(M, N) \cong \operatorname{Hom}_{A}(M, N) \\
& \underline{E x t_{A}^{i}}(M, N) \cong E x t_{A}^{i}(M, N) .
\end{aligned}
$$

Es importante entender la acción del funtor $\underline{\operatorname{Hom}}_{A}(-, A)$ en funciones entre módulos libres graduados. Si $N$ es un módulo graduado sobre el álgebra finitamente graduada $A$, entonces $\underline{\operatorname{Hom}}_{A}(N, A)$ es un $A$-módulo izquierdo vía $[a \cdot \psi](x)=a \psi(x)$. Dado un homomorfismo de $A$-módulos graduados $\phi: N_{1} \rightarrow N_{2}$, entonces induce el homomorfismo de $A$-módulos

$$
\begin{array}{ccc}
\underline{\operatorname{Hom}}_{A}\left(N_{2}, A\right) & \rightarrow & \underline{\operatorname{Hom}}_{A}\left(N_{1}, A\right) \\
f & \mapsto & f \circ \phi
\end{array}
$$

A continuación presentamos algunos resultados preliminares que se usan en la demostración de la Proposición 24.

Lema 21 ([21], Lema 1.28). Sea A un álgebra finitamente graduada.

(i) Para cada módulo libre graduado $\bigoplus_{i=1}^{m} A\left(-s_{i}\right)$, existe un isomorfismo de A-módulos graduados canónico

$$
\underline{\operatorname{Hom}}_{A}\left(\bigoplus_{i=1}^{m} A\left(-s_{i}\right), A\right) \cong \bigoplus_{i=1}^{m} A\left(s_{i}\right) .
$$

(ii) Sea $P=\bigoplus_{i=1}^{m} A\left(-s_{i}\right)$ y $Q=\bigoplus_{j=1}^{n} A\left(-t_{j}\right)$. Dado un homomorfismo de A-módulos graduados $\phi: P \rightarrow Q$, representado por la multiplicación por la matriz $M$, entonces aplicando $\mathrm{Hom}_{A}(-, A)$ se obtiene un homomorfismo de módulos $\phi^{*}: \underline{\operatorname{Hom}}_{A}(Q, A) \rightarrow \underline{\operatorname{Hom}}_{A}(P, A)$, el cual puede ser identificado canónicamente con una función de módulos graduados $\phi^{*}: \bigoplus_{j=1}^{n} A\left(t_{j}\right) \rightarrow \bigoplus_{i=1}^{m} A\left(s_{i}\right)$, usando (i). Entonces $\phi^{*}$ es definido bajo la multiplicación por la misma matriz $M$. 
Proposición 22 ([21], Proposición 1.30). Sea A un álgebra finitamente graduada. Entonces

$$
\operatorname{rgld}(A)=\operatorname{pd}\left({ }_{A} \mathbb{K}\right)=\operatorname{pd}\left(\mathbb{K}_{A}\right)=\operatorname{lgd}(A),
$$

$y$ este número es igual a la longitud de la resolución minimal libre graduada de ${ }_{A} \mathbb{K}$.

Proposición 23 ([24], Proposición 3.5). Sea $A=\sigma(R)\left\langle x_{1}, \ldots, x_{n}\right\rangle$ una extensión PBW torcida graduada de un álgebra conexa $R$. Si $R$ es noetheriana con dimensión global finita graduada, entonces A tiene dimensión global finita graduada $y \operatorname{GKdim}(A)<\infty$.

\subsection{El plano de Jordan como un álgebra Artin-Schelter regular}

En [4] fueron clasificadas las álgebras Artin-Schelter regulares de dimensión global 3 y afirman que las únicas álgebras Artin-Schelter regulares de dimensión global 2 son el plano cuántico y el plano de Jordan. En esta subsección presentamos una demostración detallada de la propiedad Artin-Schelter regular del plano de Jordan, usando el hecho que el plano de Jordan se puede expresar como una extensión PBW torcida graduada (Proposición 13).

Proposición 24. El plano de Jordan es un álgebra Artin-Schelter regular de dimensión global 2.

Demostración. Sea $A=\mathbb{K}\langle x, y\rangle /\left(y x-x y-x^{2}\right)$ el plano de Jordan y consideremos la resolución de $\mathbb{K}$ dada en (4)

$$
{ }_{A} A \stackrel{d_{2}}{\longrightarrow}{ }_{A} A^{\oplus 2} \stackrel{d_{1}}{\longrightarrow}{ }_{A} A \stackrel{j}{\longrightarrow}{ }_{A} \mathbb{K} \longrightarrow 0,
$$

donde $d_{2}(a)=a\left[\begin{array}{ll}y-x & -x\end{array}\right]$ y $d_{1}(b, c)=$ $\left[\begin{array}{ll}b & c\end{array}\right]\left[\begin{array}{l}x \\ y\end{array}\right]$, para todo $a, b, c \in A$.

Sea $A_{n}=\left\{k x_{d_{1}} x_{d_{2}} \cdots x_{d_{n}} \mid k \in \mathbb{K}, 1 \leq d_{i} \leq 2\right\}$, entonces $A=\mathbb{K} \oplus A_{1} \oplus A_{2} \oplus \cdots$.

Nótese que:

- $\operatorname{Im}\left(d_{1}\right)=\left\{b_{1} x+b_{2} y \mid b_{i} \in A\right\} \subseteq A_{\geq 1}$, entonces $\operatorname{Im}\left(d_{1}\right) \subset A_{\geq 1} A$.
- $\operatorname{Im}\left(d_{2}\right)=\left\{\left[\begin{array}{ll}a y-a x & -a x\end{array}\right] \mid a \in A\right\} \subseteq$ $A_{\geq 1} A^{\oplus 2}$, entonces $\operatorname{Im}\left(d_{2}\right) \subset A_{\geq 1} A^{\oplus 2}$.

Por la Proposición 18, se sabe que la resolución para ${ }_{A} \mathbb{K}$ es minimal. Así, de la Proposición 22 se sigue que la dimensión global de A es 2 .

Aplicando el funtor $\underline{\operatorname{Hom}}_{A}(-, A)$ a la resolución minimal de $\mathbb{K}$, obtenemos la sucesión

$$
\begin{gathered}
0 \longrightarrow \underline{\operatorname{Hom}}_{A}\left({ }_{A} \mathbb{K}, A\right) \stackrel{j^{*}}{\longrightarrow} \underline{\operatorname{Hom}}_{A}(A, A) \\
\stackrel{d_{1}^{*}}{\longrightarrow} \underline{\operatorname{Hom}}_{A}\left(A^{2}, A\right) \stackrel{d_{2}^{*}}{\longrightarrow} \underline{\operatorname{Hom}}_{A}(A, A) \longrightarrow 0 ;
\end{gathered}
$$

por el Lema 21 (i), esta última puede reescribirse como

$$
A \stackrel{d_{1}^{*}}{\longrightarrow} A^{\oplus 2} \stackrel{d_{2}^{*}}{\longrightarrow} A \longrightarrow 0,
$$

donde $d_{1}^{*}(a)=\left[\begin{array}{l}x \\ y\end{array}\right] a \quad$ y $\quad d_{2}^{*}\left(\left[\begin{array}{l}a \\ b\end{array}\right]\right)=$ $\left[\begin{array}{ll}y-x & -x\end{array}\right]\left[\begin{array}{l}a \\ b\end{array}\right]$, para todo $a, b \in A$.

En consecuencia

$$
\begin{aligned}
& \underline{\operatorname{Ext}} t_{A}^{1}(\mathbb{K}, A)=\operatorname{ker}\left(d_{2}^{*}\right) / \operatorname{Im}\left(d_{1}^{*}\right) \quad \text { y } \\
& \underline{\operatorname{Ext}} t_{A}^{2}(\mathbb{K}, A)=A / \operatorname{Im}\left(d_{2}^{*}\right) .
\end{aligned}
$$

Veamos que $\operatorname{Im}\left(d_{2}^{*}\right)=A_{\geq 1}$ : la contenencia $\operatorname{Im}\left(d_{2}^{*}\right) \subseteq A_{\geq 1}$ es inmediata. Sea $a \in A_{\geq 1}$, entonces $a$ puede escribirse de la forma $a=x a_{1}+y a_{2}$, con $a_{1}, a_{2} \in A$. Sea $\left[\begin{array}{c}a_{2} \\ -a_{1}-a_{2}\end{array}\right] \in A^{\oplus 2}$. Tenemos

$$
\begin{gathered}
d_{2}^{*}\left(\left[\begin{array}{c}
a_{2} \\
-a_{1}-a_{2}
\end{array}\right]\right)=\left[\begin{array}{ll}
y-x & -x
\end{array}\right]\left[\begin{array}{c}
a_{2} \\
-a_{1}-a_{2}
\end{array}\right] \\
=y a_{2}-x a_{2}+x a_{1}+x a_{2}=a,
\end{gathered}
$$

por tanto $A_{\geq 1} \subseteq \operatorname{Im}\left(d_{2}^{*}\right)$, con lo que $\underline{E x t}_{A}^{2}(\mathbb{K}, A)=A / A_{\geq 1} \cong \mathbb{K}$. De la Proposición 20 se tiene que $\operatorname{Ext}_{A}^{2}(\mathbb{K}, A) \cong \mathbb{K}$.

Por otra parte, note que $\left[\begin{array}{ll}y-x & -x\end{array}\right]\left[\begin{array}{l}x a \\ y a\end{array}\right]=$ $\left(y x-x y-x^{2}\right) a$ para todo $a \in A$, de donde se sigue que $\operatorname{Im}\left(d_{1}^{*}\right) \subseteq \operatorname{ker}\left(d_{2}^{*}\right)$.

Sea $\left[\begin{array}{ll}a & b\end{array}\right] \in \operatorname{ker}\left(d_{2}^{*}\right)$, entonces $y a-x a-x b=$ $\left(y x-x y-x^{2}\right) f$ para algún $f \in A$. Luego $a=x f$ y $b=y f$, por lo cual $\left[\begin{array}{ll}a & b\end{array}\right]=\left[\begin{array}{ll}x f & y f\end{array}\right] \in \operatorname{Im}\left(d_{1}^{*}\right)$. Así, $\operatorname{Im}\left(d_{1}^{*}\right)=\operatorname{ker}\left(d_{2}^{*}\right)$ y además $\operatorname{Ext}_{A}^{1}(\mathbb{K}, A)=0$. 
Finalmente, $\quad \operatorname{Ext}_{A}^{0}(\mathbb{K}, A)=\operatorname{Hom}_{A}\left({ }_{A} \mathbb{K}, A\right)=$ $\operatorname{Im}\left(j^{*}\right)=\operatorname{ker}\left(d_{1}^{*}\right)=0$.

Para concluir que el plano de Jordan es un álgebra Artin-Schelter regular, hace falta ver que tiene dimensión Gelfand-Kirillov finita. Considérese a $R=\mathbb{K}[x] \cong \mathbb{K}\langle x, y\rangle /(y)$, por la Proposición 15 se tiene que la sucesión de $\mathbb{K}[x]$-módulos proyectivos,

$$
R \stackrel{\left[\begin{array}{ll}
0 & 1
\end{array}\right]}{\longrightarrow} R^{\oplus 2} \stackrel{\left[\begin{array}{l}
x \\
y
\end{array}\right]}{\longrightarrow} R \stackrel{j}{\longrightarrow} \mathbb{K} \longrightarrow 0,
$$

es exacta. Además, como $\mathrm{R}$ es un dominio se tiene una resolución proyectiva de longitud 2. Por tanto la dimensión proyectiva de $\mathbb{K}$ como $\mathrm{R}$-módulo es menor o igual a 2. Por la Proposición 22, se tiene que $\mathbb{K}[x]$ tiene dimensión global finita. Dado que $A=\mathbb{K}\langle x, y\rangle /\left(y x-x y-x^{2}\right)$ es una extensión PBW torcida graduada de $\mathbb{K}[x]$, donde $\mathbb{K}[x]$ tiene dimensión global finita, se sigue de la Proposición 23 que $G K \operatorname{dim}(A)<\infty$. Por tanto el plano de Jordan es un álgebra Artin-Schelter regular de dimensión global 2.

Notemos que el plano de Jordan tiene la misma serie de Hilbert que el anillo de en polinomios en dos variables, lo cual implica, en particular, que $\operatorname{GKdim}(A)=2$ (véase [15]).

\section{3 Álgebras Calabi-Yau torcidas}

Si $A$ es un álgebra, el álgebra opuesta $A^{\circ}$ es el álgebra tal que $A^{\circ}=A$ como $\mathbb{K}$-módulo con producto $a * b:=b a$.

Un $A$ - $B$-bimódulo $M$ puede ser identificado como un $A \otimes B^{\circ}$-módulo izquierdo, definiendo

$$
(a \otimes b) \cdot m=a m b, \quad a \in A, b \in B, m \in M .
$$

El álgebra envolvente de $A$ es $A^{e}:=A \otimes A^{\circ}$. Como $A$ es naturalmente un $A$-bimódulo, entonces $A$ es un $A^{e}$-módulo.

Sea $M$ un $A$-bimódulo, $v, \mu: A \longrightarrow A$ dos automorfismos, el $A$-módulo torcido ${ }^{v} M^{\mu}$ es igual a $M$ como un $\mathbb{K}$-espacio vectorial con $a \cdot m \cdot b:=$ $v(a) m \mu(b)$. Así, $M$ es un $A^{e}$ - módulo con el producto dado por

$$
(a \otimes b) \cdot m=a \cdot m \cdot b=v(a) m \mu(b) .
$$

Definición 25. Una $\mathbb{K}$-álgebra $A$ es llamada $v$-Calabi-Yau torcida de dimensión $d$, para algún automorfismo $v$ de $A$ y para algún entero $\mathrm{d} \geq 0$, si se cumplen las siguientes dos condiciones:

(i) A es homológicamente suave; es decir, $A$ tiene una resolución finita de A-bimódulos proyectivos finitamente generados;

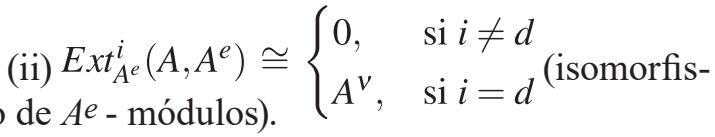

La condición (ii) es llamada la condición Calabi-Yau torcida. En este caso $v$ es llamado el automorfismo de Nakayama de A. Si el automorfismo $v$ es interno, es decir, $v(x)=a^{-1} x a$, para algún elemento invertible $a \in A$, se dice que $A$ es Calabi-Yau.

Ejemplo 26. $A=\mathbb{K}[x]$ es un álgebra Calabi-Yau. En efecto: una base como espacio vectorial de $A^{e}=A \otimes A^{o}=A \otimes A$ es el conjunto $\left\{x^{i} \otimes x^{j} \mid\right.$ $i, j \geq 0\}$. Consideremos la siguiente sucesión

$$
\begin{array}{llc}
\mathbb{K}[x] \otimes \mathbb{K}[x] & \stackrel{\mu}{\longrightarrow} & \mathbb{K}[x] \\
p(x) \otimes q(x) & \mapsto & p(x) q(x)
\end{array} \quad \longrightarrow \quad 0
$$

donde $\mu$ denota la multiplicación en el álgebra $A$. Note que esta resulta ser exacta dado que $\mu$ es un homomorfismo sobreyectivo.

Sea $\Gamma=\sum_{i=0}^{n} p_{i} \otimes q_{i} \in \operatorname{ker}(\mu)$, entonces $\sum_{i=0}^{n} p_{i}(x) q_{i}(x)=0$. Como $(1 \otimes x)^{j}=1 \otimes x^{j}$,

$$
\begin{aligned}
\Gamma & =\sum_{i=0}^{n} p_{i} \otimes q_{i}-\sum_{i=0}^{n} p_{i}(x) q_{i}(x) \otimes 1 \\
& =\sum_{i=0}^{n}\left(p_{i} \otimes q_{i}-p_{i} q_{i} \otimes 1\right) \\
& =\sum_{i=0}^{n} p_{i}\left(1 \otimes q_{i}-q_{i} \otimes 1\right) \\
& =\sum_{i=0}^{n} p_{i}\left(1 \otimes \sum_{j} b_{j}^{i} x^{j}-\sum_{j} b_{j}^{i} x^{j} \otimes 1\right)
\end{aligned}
$$




$$
\begin{aligned}
& =\sum_{i=0}^{n} p_{i} \sum_{j} b_{j}^{i}\left(1 \otimes x^{j}-x^{j} \otimes 1\right) \\
& =\sum_{i=0}^{n} p_{i} \sum_{j} b_{j}^{i}\left((1 \otimes x)^{j}-(x \otimes 1)^{j}\right) \\
& =((1 \otimes x)-(x \otimes 1)) r
\end{aligned}
$$

donde $r=\left(\sum_{i} p_{i} \sum_{j} b_{j}^{i} \sum_{t=1}^{j-1}(1 \otimes x)^{j-t}(x \otimes 1)^{t}\right)$.

Por lo tanto, $\operatorname{ker}(\mu)=(1 \otimes x-x \otimes 1)=$ $(x \otimes 1-1 \otimes x)$.

Sean $\quad P_{0}=\mathbb{K}[x] \otimes \mathbb{K}[x], P_{1}=\mathbb{K}[x] \otimes \mathbb{K}[x] \quad \mathrm{y}$ considere el siguiente homomorfismo

$$
\begin{array}{cccc}
d: & P_{1} & \longrightarrow & P_{0} \\
1 \otimes 1 & \mapsto & x \otimes 1-1 \otimes x .
\end{array}
$$

Se tiene entonces la sucesión exacta corta

$$
0 \longrightarrow \mathbb{K}[x] \otimes \mathbb{K}[x] \stackrel{d}{\longrightarrow} \mathbb{K}[x] \otimes \mathbb{K}[x] \stackrel{\mu}{\longrightarrow} \mathbb{K}[x] \longrightarrow 0 .
$$

Aplicando el funtor $\operatorname{Hom}_{A^{e}}\left(-, A^{e}\right)$, se obtiene el complejo

$$
\begin{gathered}
0 \longrightarrow \operatorname{Hom}_{A^{e}}\left(\mathbb{K}[x], A^{e}\right) \stackrel{\mu^{*}}{\longrightarrow} \operatorname{Hom}_{A^{e}}\left(P_{0}, A^{e}\right) \\
\stackrel{d^{*}}{\longrightarrow} \operatorname{Hom}_{A^{e}}\left(P_{1}, A^{e}\right) \longrightarrow 0
\end{gathered}
$$

Sea $h \in \operatorname{ker}\left(d^{*}\right)$, es decir $h \circ d=0$, donde

$$
\begin{array}{ccc}
P_{1} & \stackrel{d}{\longrightarrow} & P_{0} \\
1 \otimes 1 & \mapsto & x \otimes 1-1 \otimes x
\end{array} \quad \stackrel{h}{\longrightarrow} A^{e} .
$$

Así, $0=d^{*}(h)(1 \otimes 1)=h(d(1 \otimes 1))=h(x \otimes 1$ $-1 \otimes x)$, luego $h(x \otimes 1)=h(1 \otimes x)$ por tanto $h=0$.

Entonces $\operatorname{ker}\left(d^{*}\right)=\{0\}$.

Ahora,

$$
\begin{gathered}
\operatorname{Ext}_{A^{e}}^{0}\left(A, A^{e}\right)=\operatorname{Hom}_{A^{e}}\left(\mathbb{K}[x], A^{e}\right) \cong \operatorname{Im}\left(\mu^{*}\right) \\
=\operatorname{ker}\left(d^{*}\right)=0 . \\
\operatorname{Ext}_{A^{e}}^{1}\left(A, A^{e}\right)=\operatorname{Hom}_{A^{e}}\left(P_{1}, A^{e}\right) / \operatorname{Im}\left(d^{*}\right) \\
=\operatorname{Hom}_{A^{e}}\left(A^{e}, A^{e}\right) / \operatorname{Im}\left(d^{*}\right) \\
\cong A^{e} / \operatorname{Im}\left(d^{*}\right)=A^{e} /(x \otimes 1-1 \otimes x) .
\end{gathered}
$$

Si $\alpha \in A^{e}$, entonces $\alpha=\sum a_{i j} x^{i} \otimes x^{j}$, luego $\bar{\alpha}=\overline{\sum a_{i j} x^{i} \otimes x^{j}}$ en $A^{e} /(x \otimes 1-1 \otimes x), \quad$ por tanto $\bar{\alpha}=\sum a_{i j} \overline{x^{i+j} \otimes 1}$.

Por lo tanto,

$$
\begin{array}{rlc}
\varphi: \quad A^{e} /(x \otimes 1-1 \otimes x) & \longrightarrow & A \\
\bar{\alpha}=\sum a_{i j} x^{i+j} \otimes 1 & \mapsto & \sum a_{i j} x^{i+j}
\end{array}
$$

es un isomorfismo de módulos y se sigue que $\operatorname{Ext}_{A^{e}}^{1}\left(A, A^{e}\right) \cong A$.

Para $i \geq 2$ se tiene que $\operatorname{Ext}_{A^{e}}^{i}\left(A, A^{e}\right)=0$.

En consecuencia, el anillo de polinomios $\mathbb{K}[x]$ es un álgebra Calabi-Yau torcida de dimensión 1, con automorfismo de Nakayama $v=$ id, y como este automorfismo es trivialmente un automorfismo interno, se sigue que $\mathbb{K}[x]$ es Calabi-Yau.

\subsection{El automorfismo de Nakayama del plano de Jordan}

Como el plano de Jordan es una álgebra finitamente graduada (Proposición 2) y Artin-Shelter regular (Proposición 24), es inmediato que esta resulta ser también Calabi-Yau torcida ([20, Lema 1.2]). El objetivo de esta última subsección es usar lo hecho atrás para mostrar que, en efecto, el plano de Jordan es Calabi-Yau torcida de dimensión 2 , y calcular efectivamente su automorfismo de Nakayama.

Teorema 27 ([17], Teorema 3.3). Sea R un álgebra y $A=R[x ; \sigma, \delta]$ una extensión de Ore. Suponga que $R$ es $v$-Calabi-Yau torcida de dimensión $d$. Entonces A es Calabi-Yau torcida de dimensión $d+1$ y el automorfismo de Nakayama $v^{\prime}$ de $A$ satisface que $\left.v^{\prime}\right|_{R}=\sigma^{-1} v$ y $v^{\prime}(x)=u x+b$ con $u, b \in R$ y u invertible.

Observación 28 ([17], Observación 3.4). Si en el Teorema 27 tomamos $\sigma=$ id se sigue que $v^{\prime}(x)=$ $x+b$. Por otra parte, si $\delta=0$ entonces $v^{\prime}(x)=u x$.

Observación 29 ([11], Página 338). Sea A un álgebra $\mathbb{N}$-graduada, generada en grado uno. Si A es Artin-Schelter regular de dimensión global 2 , entonces $A \cong \mathbb{K}\left\langle x_{1}, \ldots, x_{n}\right\rangle /(f)$, donde $(f)$ es el ideal bilátero generado por el elemento $f$, con $f$ 
de la forma $f=\left[x_{1}, \ldots, x_{n}\right] M\left[x_{1}, \ldots, x_{n}\right]^{t} M$ es una matriz invertible con entradas en $\mathbb{K}$.

Aunque en [17] se presenta el automorfismo de Nakayama de una extensión de Ore de un álgebra Calabi-Yau torcida, en su demostración no es posible obtener una forma para calcularlo, por esta razón el Teorema 30 nos ayuda a calcular este automorfismo en el caso específico en que el álgebra sea el cociente entre un álgebra libre y el ideal bilátero generado por un polinomio.

Teorema 30 ([11], Proposición 3). Sean $A=$ $\mathbb{K}\left\langle x_{1}, \ldots, x_{n}\right\rangle /(f)$ y $M$ como en la Observación 29. Entonces Ext ${ }_{A^{e}}^{i}\left(A, A^{e}\right)=0$ para $i \neq 2, y$

$$
\operatorname{Ext}_{A^{e}}^{2}\left(A, A^{e}\right) \cong A^{v}(-2)
$$

donde $v$ es un automorfismo definido por $v(g)=$ $-\left[x_{1}, \ldots, x_{n}\right] M^{t} M^{-1}\left[a_{1}, \ldots, a_{n}\right]^{t}$, para $g=a_{1} x_{1}+$ $\cdots+a_{n} x_{n}$.

Nótese que si en el teorema anterior $A$ es $\mathrm{Ca}$ labi-Yau torcida de dimensión 2, entonces el automorfismo $v$ es el automorfismo de Nakayama de $A$.

Proposición 31. El plano de Jordan es un álgebra Calabi-Yau torcida, con automorfismo de Nakayama $v$ dado por $v(x)=x y v(y)=2 x+y$.

Demostración. Sea $A=\mathbb{K}\langle x, y\rangle /\left(y x-x y-x^{2}\right)$ el plano de Jordan. Por la Proposición 6 el plano de Jordan es una extensión de Ore de $\mathbb{K}[x]$, con $\sigma(x)=x$ y $\delta(x)=x^{2}$. Como $\mathbb{K}[x]$ es Calabi-Yau (en particular es Calabi-Yau torcida), entonces del Teorema 27 se tiene que el plano de Jordan es un álgebra Calabi-Yau torcida. Como $\sigma=i_{\mathbb{K}[x]}$, entonces por la Observación 28 se tiene que el automorfismo de Nakayama de A es de la forma $v(x)=x \mathrm{y} v(y)=y+p \operatorname{con} p \in \mathbb{K}[x]$.

Sea $f=y x-x y-x^{2}$, entonces $f$ puede ser reescrito de la forma $f=\left[\begin{array}{ll}x & y\end{array}\right]\left[\begin{array}{cc}-1 & -1 \\ 1 & 0\end{array}\right]\left[\begin{array}{l}x \\ y\end{array}\right]$. Por el Teorema 30, dado $p(x, y)=a x+b y \in A$, se tiene que

$$
\begin{gathered}
v(p(x, y))=-\left[\begin{array}{ll}
x & y
\end{array}\right]\left[\begin{array}{ll}
-1 & 1 \\
-1 & 0
\end{array}\right]\left[\begin{array}{cc}
0 & 1 \\
-1 & -1
\end{array}\right]\left[\begin{array}{l}
a \\
b
\end{array}\right] \\
=x a+(2 x+y) b .
\end{gathered}
$$

Como $v$ es un automorfismo de $A$, necesariamente $\quad v(k)=k$, para todo $k \in \mathbb{K}$. Pero $x=1 x+0 y$, entonces $v(x)=x+(2 x+y) 0=x$, $\mathrm{y}$ al ser $y=0 x+1 y$, se sigue que $v(y)=x 0+$ $(2 x+y) 1=2 x+y$.

Notemos que el plano de Jordan no es Calabi-Yau, pues $v$ no es un automorfismo interno.

Observación 32. Si $A$ es un álgebra Calabi-Yau torcida, entonces $A$ puede ser extendida a un álgebra Calabi-Yau, es decir, si $A$ es Calabi-Yau torcida con automorfismo de Nakayama $\sigma$, entonces $A[z ; \sigma]$ es Calabi-Yau (véase $[9$, Teorema 1.1 y Observación 5.1]).

Ejemplo 33. Sea $A$ el plano de Jordan con automorfismo de Nakayama $v$ como en la Proposición 31 , entonces

$$
B=A[z ; v]=\mathbb{K}[x][y ; \delta][z ; v]
$$

es una extensión de Ore de $A$. Por Teorema 27, $B$ es Calabi-Yau torcida con automorfismo de Nakayama $v^{\prime}$ tal que $v^{\prime}(x)=x$ y $v^{\prime}(y)=y$ y podemos escribir el álgebra $B$ como $B=\mathbb{K}[x, z][y ; \delta]$ donde $\delta(x)=x^{2}$. Por otro lado, en $B=A[z ; v]$ tenemos que $z y=v(y) z=(y+2 x) z=y z+2 x z$, mientras que en $B=\mathbb{K}[x, z][y ; \delta]$ vale $y z=z y+\delta(z)$. Así, $y z-\delta(z)=2 x z+y z$ y por lo tanto, $\delta(z)=-2 x z$. Entonces, $y z=z y-2 x z=v^{\prime}(z) y+\delta(z)$, es decir, $v^{\prime}(z)=z$. Por lo tanto $v^{\prime}=i_{B}$ es un automorfismo interno y $B$ resulta ser Calabi-Yau.

\section{Agradecimientos}

Los autores expresamos nuestros agradecimientos a los (las) jurados anónimos(as) por sus valiosas sugerencias para el mejoramiento del artículo. 


\section{REFERENCIAS}

[1] N. Andruskiewitsch, I. Angiono, I. Heckenberger, "Liftings of Jordan and Super Jordan Planes", Proc. Edinb. Math. Soc., vol. 61, no. 3, pp. 661-672, 2018.

[2] N. Andruskiewitsch, I. Angiono, I. Heckenberger, "On finite GK-dimensional Nichols algebras over abelian groups", arXiv:1606.02521v2 [math.QA], 2018.

[3] N. Andruskiewitsch, D. Bagio, S. Della Flora y D. Flôres, "Representations of the super Jordan plane", São Paulo J. Math. Sci., vol. 11, no. 2, pp. 312-325, 2017.

[4] M. Artin y W. Schelter, "Graded Algebras of Global Dimension 3", Adv. Math., vol. 66, pp. 171-206, 1987.

[5] E. E. Demidov, Yu. I. Manin, E. E. Mukhin and D. V. Zhdanovich, "Nonstandard quantum deformations of GL(n) and constant solutions of the Yang-Baxter equation", Common trends in mathematics and quantum field theories (Kyo-to, 1990). Progr. Theoret. Phys. Suppl., no. 102 (1990), pp. 203-218 (1991).

[6] C. Gallego y O. Lezama, "Gröbner bases for ideals of $\sigma-\mathrm{PBW}$ extensions", Comm. Algebra, vol. 39, pp. 50-75, 2011.

[7] V. Ginzburg, "Calabi-Yau algebras", arXiv:math. $A G / 0612139 v 3$, vol. 51, pp. 329-333, 2006.

[8] N. R. González y Y. P. Suárez, "Ideales en el Anillo de Polinomios Torcidos R[x; $\sigma, \delta]$ ", Ciencia en Desarrollo, vol. 5, no. 1, pp. 3137, 2014.

[9] J. Goodman y U. Krähmer, "Untwisting a twisted Calabi-Yau algebra", J. Algebra, vol. 406, pp. 272-289, 2014.

[10] D. I. Gurevich, "The Yang-Baxter equation and the generalization of formal Lie theory", Dokl. Akad. Nauk SSSR, vol. 288, no. 4, pp. 797-801, 1986.
[11] J.W He, F. Oystaeyen y Y. Zhang, "Calabi-Yau algebras and their deformations", Bull. Math. Soc. Sci. Math. Roumanie, vol. 56, no. 3, pp. 335- 347, 2013.

[12] N. Iyudu, "Representation Spaces of the Jordan Plane", Comm. Algebra, vol. 42, no. 8, pp. 3507-3540, 2014.

[13] S. Korenskii, "Representations of the Quantum group SLj(2)", Rus. Math. Surv, vol. 46, no. 6, pp. 211-212, 1991.

[14] G. R. Krause y T. H. Lenagan, Growth of algebras and Gelfand-Kirillov dimension, Grad. Stud. Math., 22, AMS (2000).

[15] J. McConnell y J. Robson, Noncommutative Noetherian Rings, Graduate Studies in Mathematics, AMS (2001).

[16] O. Lezama y E. Latorre, "Noncommutative algebraic geometry of semigraded rings", Internat. J. Algebra Comput., vol. 27, no. 4, pp. 361-389, 2017.

[17] L. Liu, S. Wang y Q. Wu, "Twisted Calabi-Yau property of Ore extensions", J. Noncommut. Geom., vol. 8, no. 2, pp. 587-609, 2014.

[18] S. Reca y A. Solotar 2018. "Homological invariants relating the super Jordan plane to the Virasoro algebra", J. Algebra, vol. 507, pp. 120-185.

[19] A. Reyes y H. Suárez, "Some remarks about the cyclic homology of skew PBW extensions", Ciencia en Desarrollo, vol. 7, no. 2, pp. 99-107, 2016.

[20] M. Reyes, D. Rogalski y J. J Zhang, "Skew Calabi-Yau algebras and homological identi-ties", Adv. Math., vol. 264, pp. 308 -354, 2014.

[21] D. Rogalski, "An introduction to non-commutative projective algebraic geometry", arXiv:1403.3065 [math.RA], 2014. 
[22] H. Suárez, "Koszulity for graded skew PBW extensions", Comm. Algebra, vol. 45, no. 10, pp. 4569-4580, 2017.

[23] H. Suárez, O. Lezama y A. Reyes, "Some Relations between N-Koszul, Artin-Schelter Regular and Calabi-Yau with Skew PBW Extensions", Ciencia en Desarrollo, vol. 6, no. 2, pp. 205- 213, 2015.
[24] H. Suárez, O. Lezama y A. Reyes, "Calabi-Yau property for graded skew PBW extensions", Rev. Colombiana Mat., vol. 51, no. 2, pp. 221-238, 2017.

[25] H. Suárez y A. Reyes, "Koszulity for skew PBW extension over fields", JP J. Algebra Number Theory Appl., vol. 39, no. 2, pp. 181-203, 2017. 